\title{
A MOSQUITO DENSOVIRUS INFECTING AEDES AEGYPTI AND AEDES ALBOPICTUS FROM THAILAND
}

\author{
PATTAMAPORN KITTAYAPONG, KATHY J. BAISLEY, AND SCOTT L. O'NEILL \\ Department of Biology, Faculty of Science, Mahidol University, Bangkok, Thailand; Section of Vector Biology, Department of \\ Epidemiology and Public Health, Yale University School of Medicine, New Haven, Connecticut
}

\begin{abstract}
A previously undescribed mosquito densovirus was detected in colonies of Aedes aegypti and Ae. albopictus from Thailand, using a polymerase chain reaction (PCR)-based assay. Phylogenetic analysis of this virus showed it to be most closely related to ADNV isolated from Russian Ae. aegypti. Both Aedes species were susceptible to oral infection with the Thai-strain virus. Larval mortality for Ae. albopictus was higher (82\%) than for Ae. aegypti $(51 \%)$. Aedes aegypti were able to transmit the virus vertically to a high $(58 \%)$ proportion of $\mathrm{G}_{1}$ progeny, and the virus was maintained persistently for up to six generations. A PCR survey of adult Ae. aegypti and Ae. albopictus in Thailand indicated that only Ae. aegypti are infected in the field, with an overall prevalence of 44\%. Densovirus infection in adult Ae. aegypti showed distinct seasonal variation. The Thai strain densovirus may play a role in structuring Ae. albopictus and Ae. aegypti populations in nature.
\end{abstract}

Insect densoviruses are small, autonomous, non-enveloped DNA viruses that belong to the family Parvoviridae. They are characterized by the presence of inverted terminal repeat sequences and the separate encapsidation of complementary single-stranded DNA. Two subgroups of insect densoviruses have been recognized on the basis of lepidopteran isolates. ${ }^{1}$ Subgroup I densoviruses have a 6-kb genome, code their structural and nonstructural proteins from separate strands, and infect all insect cells except those of the midgut. Subgroup II densoviruses have a smaller genome, approximately $4.9 \mathrm{~kb}$, code all their proteins from one strand, and infect only insect midgut cells. Mosquito densoviruses do not easily fit into this classification scheme, and as such form a third subgroup. They are characterized as having a 4-kb genome and infecting all tissues of their insect hosts. ${ }^{2}$ While Subgroup I and II densoviruses typically encapsidate both plus and minus DNA strands with equal frequency, some mosquito densoviruses encapsidate primarily minus strands. . $^{3,4}$

To date, five mosquito densoviruses have been described. The first, ADNV, was isolated from Aedes aegypti larvae, ${ }^{3,5}$ while the second, $A a \mathrm{PV}$, was recovered from an Ae. albopictus (C6/36) cell line., ${ }^{4,6,7}$ Genomic sequence data have been obtained for both these densoviruses. A third was observed by electron microscopy of an Ae. pseudoscutellaris (AP-61) cell line. ${ }^{8}$ More recently, two additional mosquito densoviruses ( $\mathrm{TaDNV}$ and $\mathrm{HeDNV}$ ) were detected using a polymerase chain reaction (PCR) assay in infected Toxorhynchites amboinensis (TRA-284) and Haemagogus equinus (GML-HE-12) cell lines, respectively. ${ }^{2}$ The PCR products from both viruses were sequenced, and infection was further confirmed by electron microscopy and indirect fluorescent antibody tests.

Mosquito densoviruses are believed to be maintained in nature primarily by horizontal transmission, although transovarial and venereal transmission have also been recorded..$^{2,9}$ These viruses are often pathogenic to their arthropod hosts. $A \mathrm{DNV}$ was found to efficiently kill larval Ae. aegypti, as well as Ae. caspius, and to a lesser extent Culex pipiens. ${ }^{10}$ $A a \mathrm{PV}$ is highly pathogenic for Ae. aegypti, killing up to $95 \%$ of larvae after oral infection. ${ }^{4,11}$ Although TaDNV appeared nonpathogenic in experiments with Aedes and Culex, up to
$10 \%$ of Ae. aegypti larvae died after being orally infected with $\mathrm{HeDNV} .^{2}$

In this paper, we describe a sixth mosquito densovirus, designated $A T h \mathrm{DNV}$, isolated from adult Ae. albopictus and Ae. aegypti mosquitoes from Thailand. We report on the infectivity and pathogenicity of this virus in Ae. aegypti and Ae. albopictus, and its prevalence in natural populations of both species. In addition, we describe its vertical transmission and persistence in an Ae. aegypti colony.

\section{MATERIALS AND METHODS}

Virus source. The Thai strain densovirus was detected in a colony of Ae. albopictus that originated from larvae collected in 1992 from Krabi Province in southern Thailand, and had been in the laboratory for 5 generations. The virus was also detected in a colony of Ae. aegypti that was started with adult mosquitoes collected in 1993 from Chachoengsao Province in eastern Thailand, and had been in the same laboratory for 3 generations. Infected larvae from the Ae. aegypti colony were used as the source of virus in the investigations of infectivity, pathogenicity, and vertical transmission. Although it is not known how these colonies first acquired their infections, we subsequently refer to them as naturally infected to distinguish from mosquitoes that we infected experimentally.

Mosquitoes used in experimental infection studies. Three colonies of Aedes mosquitoes were used for investigations on virus infectivity and pathogenicity. Two uninfected Ae. aegypti colonies originating from geographically distant $(>800 \mathrm{~km})$ populations were established; one from larval collections made in Chachoengsao Province in 1994 (colony CHA), and the second from egg collections made in Songkhla Province, southern Thailand in 1995 (colony SON). In addition, an uninfected Ae. albopictus colony was founded with adult females collected in Chachoengsao Province in 1994. To ensure that newly colonized mosquitoes were not infected with densovirus, field-collected females were allowed to oviposit in individual vials, then were killed and screened by a PCR after oviposition. Offspring of densovirus-negative mothers were maintained together and raised through 4 generations, then tested again by the PCR before being used in the experiments. 
Detection of mosquito densoviruses by the PCR. Mosquitoes were screened with a PCR assay using primers designed from the densovirus open reading frame $3 .^{2}$ Crude DNA extractions were performed by homogenizing an individual adult or larval mosquito in $100 \mu \mathrm{l}$ of STE buffer (100 mM NaCl, 10 mM Tris-HCl, 1 mM EDTA, pH 8.0), using the methods of O'Neill and others. ${ }^{12}$ One microliter of the supernatant was used as DNA template in the PCR. Densovirus-infected Ae. albopictus C6/36 cell line DNA was used as a positive control. Viral DNA was PCR amplified in 20- $\mu$ l reaction volumes: $2 \mu \mathrm{l}$ of $10 \times$ buffer (Promega, Madison, WI), $2 \mu \mathrm{l}$ of $25 \mathrm{mM} \mathrm{MgCl} 2,0.5 \mu \mathrm{l}$ of dNTPs $(10 \mathrm{mM}$ each), $0.5 \mu \mathrm{l}$ of each primer $(20 \mathrm{mM}$ each), and 1 unit of Taq DNA polymerase (Promega). The PCR thermal profile was $95^{\circ} \mathrm{C}$ for $1 \mathrm{~min}, 55^{\circ} \mathrm{C}$ for $1 \mathrm{~min}$, and $72^{\circ} \mathrm{C}$ for $1 \mathrm{~min}$ per cycle for 35 cycles. The PCR products were run on a $1 \%$ agarose gel with a 1-kb ladder (Gibco/BRL, Gaithersburg, MD) to determine the presence and size of amplified DNA. Samples that yielded products of the expected size (350 basepairs) were scored as positive. The Thai strain densovirus was initially detected by PCR screening of adult mosquitoes from two colonies of Aedes. To further confirm the presence of densovirus in these colonies, amplified products were digested with Ava II since a Ava II restriction site was always present in all known mosquito densoviruses. Digestion with Ava II yields products of approximately 110 and 240 basepairs.

Phylogenetic analysis of $\boldsymbol{A T h D N V}$ fragments. To characterize the genome of the Thai densovirus, DNA samples from the naturally infected Ae. albopictus and Ae. aegypti colonies were amplified and sequenced. The PCR products were purified using spin columns (Wizard PCR Preps; Promega) and sequenced on an ABI377 automated sequencer (Perkin Elmer, Norwalk, CT). Resulting sequences were manually aligned with other described mosquito densovirus sequences and subjected to maximum parsimony analysis using the heuristic search algorithm of PAUP version 3.0. ${ }^{13}$ One hundred bootstrap replications were carried out. The number of nucleotide substitutions was estimated using the two-parameter method ${ }^{14}$ with PHYLIP version $3.5 \mathrm{c} .{ }^{15}$

Susceptibility to $A T h \mathrm{DNV}$ and pathogenicity for $A e$. aegypti and Ae. albopictus. Pools of 50 first-instar larvae from the uninfected Ae. aegypti (CHA) and Ae. albopictus colonies were placed in separate autoclaved rearing trays with 1 liter of distilled water, with 2 replicates for each species. Approximately 20 freshly dead larvae from the naturally infected Ae. aegypti colony were confirmed infected by PCRscreening of larval heads, then ground and added to each rearing tray as a source of densovirus. Mosquito larvae were reared to adulthood under insectary conditions, and fed on autoclaved fish food pellets until pupation. Surviving adults were killed and assayed by the PCR to compare the densovirus infection rate in adult Ae. aegypti and Ae. albopictus mosquitoes.

Susceptibility to infection between two populations of Ae. aegypti. Pools of 100 first-instar larvae from each of the two uninfected Ae. aegypti colonies were placed in separate autoclaved rearing trays with 1 liter of distilled water, with 2 replicates for each population. Approximately 20 PCRpositive dead larvae from the naturally infected Ae. aegypti colony were ground and added to each rearing tray as a source of densovirus. Mosquito larvae were reared to adulthood as previously described. Surviving adults were assayed by the PCR to compare susceptibility to the Thai strain densovirus between two geographically distant Ae. aegypti populations.

Vertical transmission and persistence of $A T h D N V$ in Ae. aegypti. Adult female Ae. aegypti from the naturally infected colony were used to establish 14 infected female lines. Parent generation $\left(\mathrm{G}_{0}\right)$ females were bloodfed and individually isolated for egg-laying. After oviposition, females were killed and tested by the PCR to confirm infection status. The $G_{1}$ eggs from each densovirus-positive female were hatched and reared separately in autoclaved trays as described above. Dead larvae and pupae were removed daily. Adult $G_{1}$ offspring from each infected female line were maintained in separate cages and allowed to mate with their siblings. One week after emergence, $G_{1}$ females were blood fed and allowed to oviposit on common egg paper set up in each cage, then screened by the PCR for the presence of densovirus. Successive generations from each female line were maintained together in the same manner for 7 generations, with at least 10 offspring from each female-line generation being tested for densovirus.

Prevalence of $A T h \mathrm{DNV}$ in natural Ae. aegypti and $A e$. albopictus populations. Adult Ae. albopictus and Ae. aegypti from 11 provinces throughout Thailand were collected using mosquito landing catches and attractant resting boxes, ${ }^{16,17}$ from January to June 1997. Specimens were identified using morphologic keys, ${ }^{18,19}$ and stored at $-70^{\circ} \mathrm{C}$ until DNA was extracted. Mosquitoes were screened by the PCR for the presence of densovirus as previously described. In addition, seasonal variation in densovirus infection prevalence in $A e$. aegypti was investigated. Mosquitoes were collected from 12 randomly selected houses in Village 2, Plaeng Yao District, Chachoengsao Province from April to September 1995, using attractant resting boxes. Specimens were identified using morphologic keys and stored at $-70^{\circ} \mathrm{C}$ until screened by the PCR for the presence of densovirus.

Statistical analysis. The significance of differences in densovirus infection frequency between mosquito groups was evaluated with chi-square and Mann-Whitney U tests as indicated, using Statistix Version 4.1 (Analytical Software, Tallahassee, FL). Filial infection rates among successive generations were compared by one-way analysis of variance (ANOVA), with proportions arcsin transformed. Spearman's rank correlation coefficient was used to investigate the association between vertical transmission and mortality rates. Probability values $<0.05$ were considered significant.

\section{RESULTS}

Detection and characterization of $A T h D N V$ from $A e$. albopictus and Ae. aegypti. The PCR screening using densovirus specific primers revealed the presence of the viral genome in laboratory colonies of Ae. albopictus and Ae. aegypti. Products of the expected size (350 basepairs) were amplified from both species. Amplified products could be digested with Ava II to fragments approximately 110 and 240 basepairs in size, further confirming their identity as densovirus DNA. The densovirus sequences obtained from both infected Aedes colonies were identical at the DNA lev- 


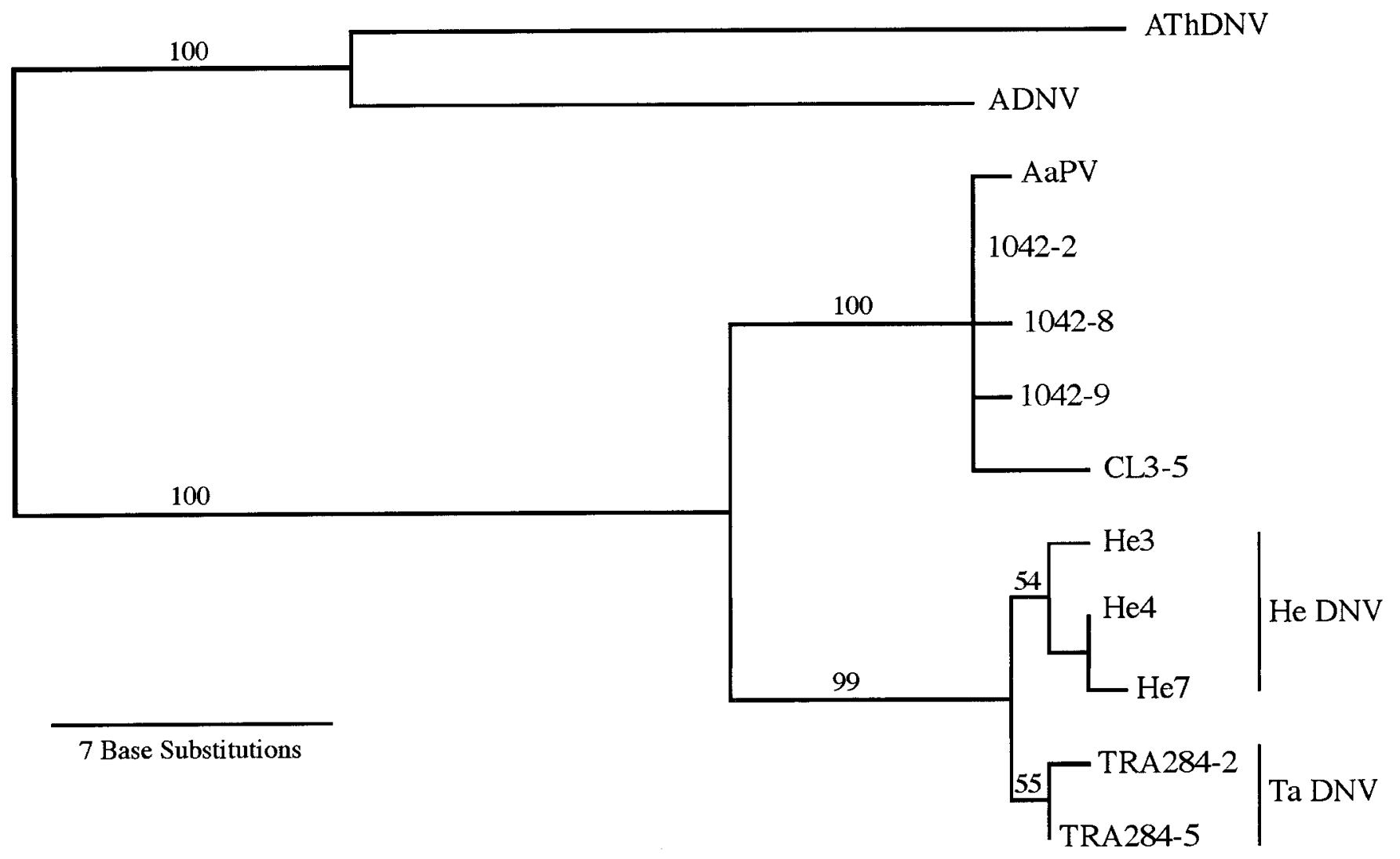

FIGURE 1. Most parsimonious unrooted tree generated from heuristic search algorithm of PAUP version 3.0 based on 301 basepairs of sequence data, showing the relationship of $A T h \mathrm{DNV}$ with other described mosquito densoviruses. Numbers above the branches refer to bootstrap values for 100 replicates. Source of nucleotide sequence data is as follows: ADNV from Aedes aegypti (GenBank accession no. M37899); $A a \mathrm{PV}$ from an Ae. albopictus cell line (GenBank accession no. X74945); 1042-2, 1042-8, and 1042-9 from an Ae. aegypti cell line; ${ }^{2}$ CL3-5 from a Culex theileri cell line $;{ }^{2} \mathrm{He} 3, \mathrm{He} 4, \mathrm{He} 7$ from a Haemagogus equinus cell line; ${ }^{2}$ and TRA284-2 and TRA284-5 from a Toxorhynchites amboinensis cell line. ${ }^{2}$

el. These sequences could be easily aligned with those of other known densoviruses. The Thai densovirus most closely resembled $A D N V,{ }^{3}$ with a $13.3 \%$ divergence at the DNA level (Kimura 2-parameter distance, transition:transversion ratio of 1:2). Divergence from other known mosquito densoviruses ranged between $20.9 \%$ and $22.3 \%$. Maximum parsimony analysis generated one most parsimonious tree (Figure 1) and upheld the results of the nucleotide similarity data. Bootstrap values supported all major branches of the phylogenetic tree, which separated mosquito densoviruses into three main groups.

Aedes susceptibility to infection with AThDNV. Eighty percent of surviving Ae. aegypti adults (20 of 25) were PCR positive for densovirus after first-instar larval infection with AThDNV (Table 1). In contrast, 33.3\% (6 of 18) of surviving Ae. albopictus adults appeared infected. Larval mortality in

TABLE 1

First-instar oral infectivity of $A T h \mathrm{DNV}$ for mosquitoes as measured by positive polymerase chain reaction amplification

\begin{tabular}{lcc}
\hline Mosquito species & $\begin{array}{c}\text { Infection in } \\
\text { surviving adults }\end{array}$ & Mortality \\
\hline Aedes aegypti & $80.0 \%(20 / 25)$ & $51.0 \%(51 / 100)$ \\
Ae. albopictus & $33.3 \%(6 / 18)$ & $82.0 \%(82 / 100)$ \\
\hline
\end{tabular}

Ae. albopictus was $82 \%$ (82 of 100) compared with $51 \%$ (51 of 100) in Ae. aegypti, suggesting that the virus may be more pathogenic to Ae. albopictus.

Both colonies of Ae. aegypti appeared equally susceptible to horizontal infection with the virus (Table 2), with $79.4 \%$ (54 of 68) of surviving adults from the Chachoengsao colony and $71.0 \%$ (71 of 100) from the Songkhla colony being infected $\left(\chi^{2}=1.5\right.$, degrees of freedom $\left.[\mathrm{df}]=1, P>0.22\right)$. Overall infection frequencies did not vary between males and females $\left(\chi^{2}=0.10\right.$, df $\left.=1, P>0.78\right)$. Eighty percent of females (28 of 35) and $78.8 \%$ (26 of 33) of males from the Chachoengsao colony were positive for densovirus infection, compared with $71.1 \%$ (59 of 83) of females and $70.6 \%$ (12 of 17) of males from the Songkhla colony.

Vertical transmission and persistence of $A T h D N V$ in Ae. aegypti. All 14 female lines transmitted the infection to at least some of their $\mathrm{G}_{1}$ progeny. Mean adult survival in the first generation was $68.7 \%$ (167 of 243), with an average of $57.5 \%$ (96 of 167) of surviving adults being infected (Table 3). Filial infection rates in adult G1 progeny were positively associated with mortality in the same generation; this relationship was marginally significant $(\mathrm{r}=0.44, \mathrm{n}=14, P=$ 0.11 , by the Spearman rank correlation coefficient). Four female lines did not produce surviving offspring beyond the second generation. Densovirus infection was detected for 4 
TABLE 2

Oral infectivity of $A T h \mathrm{DNV}$ for Aedes aegypti as measured by positive polymerase chain reaction amplification in surviving adults

\begin{tabular}{|c|c|c|c|}
\hline Mosquito population & Total adults infected & Females infected & Males infected \\
\hline Chachoengsao & $79.4 \%(54 / 68)$ & $80.0 \%(28 / 35)$ & $78.8 \%(26 / 33)$ \\
\hline Songkhla & $71.0 \%(71 / 100)$ & $71.1 \%(59 / 83)$ & $70.6 \%(12 / 17)$ \\
\hline Total & $74.4 \%(125 / 168)$ & $73.7 \%(87 / 118)$ & $76.0 \%(38 / 50)$ \\
\hline
\end{tabular}

generations in all 10 of the remaining lines, for 5 generations in 7 lines, and for 6 generations in 4 lines. Mean filial infection rates were not significantly different among the first 4 generations, with an average of $52.8 \%$ (391 of 740) of surviving adults being infected $\left(\mathrm{F}_{3,86}=0.62, P=0.61\right.$; by ANOVA, proportions arcsin transformed). Within those female lines that continued to maintain the virus beyond the fourth generation, adult infection rates decreased to $32.7 \%$ (51 of 156) in the fifth and sixth generations. No infected adult offspring were observed by the seventh generation in any of the female lines tested. Filial infection rates showed no significant sex bias, with an average of $42.7 \%$ (234 of 548 ) of females and $42.3 \%$ (208 of 492) of males being PCR-positive for densovirus ( $P=0.59$, by the Mann-Whitney U test).

Prevalence of $A T h \mathrm{DNV}$ in natural Ae. aegypti and Ae. albopictus populations. A total of 97 field-caught adult $A e$. aegypti and 79 adult Ae. albopictus from 11 provinces throughout Thailand were screened by the PCR for densovirus (Table 4). Infection prevalence in Ae. aegypti was $44.3 \%$ (43 of 97). In contrast, none of the Ae. albopictus tested were positive for densovirus infection. To investigate seasonal variation in infection prevalence, Ae. aegypti adults were collected monthly from attractant resting boxes in houses in Village 2, Chachoengsao Province from April to September 1995. An average of $22.4 \%$ (189 of 844) of Ae. aegypti tested positive for densovirus infection by the PCR assay (Table 5). Median infection prevalence was not significantly different between males (134 of 571) and females (55 of 273) ( $P=0.26$, by the Mann-Whitney U test). Significant heterogeneity in monthly infection frequency was detected $\left(\chi^{2}=54.28\right.$, df $\left.=5, P<0.001\right)$, with the most PCR-positive mosquitoes recorded in July (108 of 308) and the fewest in August ( 2 of 31). Furthermore, no females tested positive for densovirus during the months of August ( 0 of 7 ) and September (0 of 39), when overall prevalence was the lowest.

\section{DISCUSSION}

We have detected a Thai strain densovirus in Aedes mosquitoes that appears to be distinct from other described mosquito densoviruses. Phylogenetic analysis shows it to be most closely related to the $A \mathrm{DNV}$ described by Afanasiev and others, ${ }^{3}$ with $86.7 \%$ similarity on the DNA level, based on Kimura 2-parameter distances. In addition, the Thai densovirus appears to be ecologically distinct from known mosquito densoviruses, with high rates of vertical transmission and persistence over several generations. As such, we propose a new designation, $A T h \mathrm{DNV}$, for this densovirus strain.

$A T h \mathrm{DNV}$ was initially detected in an Ae. albopictus colony that had been maintained in the laboratory for 5 generations. The identical virus was also detected in an Ae. aegypti colony that was maintained at the same facility. Our data indicate that densovirus infection is rare in natural populations of adult Ae. albopictus in Thailand. In contrast, infection is widespread and common in adult field populations of Ae. aegypti. It is likely that the AThDNV infection initially detected in the laboratory colony of Ae. albopictus was acquired by chance contamination with Ae. aegypti. Several explanations may account for the difference in infection prevalence between these two species in the field. Aedes albopictus may have less contact with the virus as a result of its larval habitat utilisation. Alternatively, infection prevalence in adult Ae. albopictus may be low as a result of high larval-stage mortality (Table 1), with few infected individuals surviving to adulthood.

Aedes aegypti and Ae. albopictus both appear to be susceptible to oral infection with AThDNV. More than $80 \%$ of surviving Ae. aegypti adults were PCR positive for densovirus after first-instar infection with the virus. The infection rate in surviving Ae. albopictus adults was much lower, approximately $30 \%$. Our data suggest that $A T h \mathrm{DNV}$ may be more pathogenic for Ae. albopictus, with more than $80 \%$ of the larvae dying before pupation, compared with a $50 \%$ lar-

TABLE 3

Vertical transmission and persistence of $A T h \mathrm{DNV}$ in naturally infected Aedes aegypti, measured by polymerase chain reaction

\begin{tabular}{lccccc}
\hline & \multirow{2}{*}{$\begin{array}{c}\text { No. of female } \\
\text { Generation }\end{array}$} & $\begin{array}{c}\text { No. of female } \\
\text { lines transmitting } \\
\text { AthDNV }\end{array}$ & \multicolumn{3}{c}{ Infection in surviving adult progeny } \\
\cline { 5 - 6 } & 14 & 14 & $57.5 \%(96 / 167)$ & Female & Male \\
2 & 11 & 11 & $48.3 \%(87 / 180)$ & $59.8 \%(55 / 92)$ & $54.7 \%(41 / 75)$ \\
3 & 10 & 10 & $52.2 \%(130 / 249)$ & $48.4 \%(41 / 74)$ & $43.4 \%(46 / 106)$ \\
4 & 10 & 10 & $54.2 \%(78 / 144)$ & $56.9 \%(41 / 76)$ & $59.6 \%(53 / 89)$ \\
5 & 10 & 7 & $20.8 \%(30 / 144)$ & $13.9 \%(10 / 72)$ & $51.4 \%(37 / 72)$ \\
6 & 7 & 4 & $19.4 \%(21 / 108)$ & $18.5 \%(10 / 54)$ & $27.8 \%(20 / 72)$ \\
7 & 4 & 0 & $0 \%(0 / 48)$ & $0 \%(0)$ & $20.4 \%(11 / 54)$ \\
Total & & & $42.5 \%$ & $42.7 \%$ & $0 \%(0 / 24)$ \\
& & & $(442 / 1040)$ & $(234 / 548)$ & $42.3 \%$ \\
& & & & & $(208 / 492)$ \\
\hline
\end{tabular}


TABLE 4

Densovirus infection prevalence in natural populations of Aedes aegypti and Ae. albopictus in Thailand, based on a polymerase chain reaction

\begin{tabular}{lll}
\hline \multicolumn{1}{c}{ Location (Province) } & Ae. aegypti & Ae. albopictus \\
\hline Chaiyaphum & $17.6 \%(3 / 17)$ & $0.0 \%(0 / 11)$ \\
Kanchanaburi & $33.3 \%(2 / 6)$ & $0.0 \%(0 / 16)$ \\
Lumpang & $33.3 \%(1 / 3)$ & $0.0 \%(0 / 3)$ \\
Lumphun & $50.0 \%(2 / 4)$ & $0.0 \%(0 / 3)$ \\
Nakhon Ratchasima & $38.9 \%(7 / 18)$ & $0.0 \%(0 / 5)$ \\
Phitsanulok & $50.0 \%(2 / 4)$ & $0.0 \%(0 / 2)$ \\
Phrae & $50.0 \%(2 / 4)$ & $0.0 \%(0 / 4)$ \\
Satun & $50.0 \%(5 / 10)$ & $0.0 \%(0 / 5)$ \\
Songkhla & $71.4 \%(10 / 14)$ & $0.0 \%(0 / 10)$ \\
Surat Thani & $100.0 \%(5 / 5)$ & $0.0 \%(0 / 10)$ \\
Ubon Ratchathani & $33.3 \%(4 / 12)$ & $0.0 \%(0 / 10)$ \\
Total & $44.3 \%(43 / 97)$ & $0.0 \%(0 / 79)$ \\
\hline
\end{tabular}

val mortality in experimentally infected Ae. aegypti. However, since viral titer was not estimated in our study, it is possible that differences in mortality between the two species were a result of differences in inoculating dose. High levels of pathogenicity have also been observed with $A a \mathrm{PV}$. Barreau and others ${ }^{11}$ noted up to $90 \%$ mortality of Ae. aegypti when ground infected larvae were added to the water in which they were reared. It is clear that the Thai strain densovirus can also be transmitted with relatively low host mortality, as indicated by its maintenance in the Aedes colonies where it was originally detected. Larval mortality in these naturally infected colonies appears to be on the order of $30 \%$ (Kittayapong P, unpublished data). Presumably these colonies encounter much lower virus concentrations under normal rearing conditions than in our experimental inoculum.

The two populations of Ae. aegypti showed a similar susceptibility to oral infection with $A T h \mathrm{DNV}$. Overall infection frequency in surviving adults was $74 \%$, and showed no significant sex bias. In their experiments with $A a \mathrm{PV}$, Barreau and others ${ }^{11}$ recorded infection rates up to $32 \%$ in $A e$. aegypti adults that had been exposed to the virus as first instar larvae. O'Neill and others ${ }^{2}$ found $21 \%$ of adult Ae. aegypti to be PCR-positive for densovirus after first-instar infection with $H e$ DNV. These infection rates in adult Ae. aegypti are less than half of those we report for the Thai densovirus strain. Our observations may be explained by a lower pathogenicity of AThDNV for Ae. aegypti or an enhanced susceptibility of $A e$. aegypti to AThDNV.

Vertical transmission has been reported with $A \mathrm{DNV}$ and $A a \mathrm{PV}$ in $A e$. aegypti, and with $H e \mathrm{DNV}$ in Ae. albopictus. ${ }^{2,9}$
O'Neill and others ${ }^{2}$ found $H e$ DNV to be vertically transmitted to $20 \%$ of adult offspring of experimentally infected $A e$. albopictus mothers; however, the virus was not transmitted in other mosquito species, including Ae. aegypti. Barreau and others ${ }^{9}$ showed that vertical transmission of $A a \mathrm{PV}$ in experimentally infected Ae. aegypti depended upon virus titer in the infected mother. Females with low virus titers produced no infected adult progeny, while those with higher titers transmitted the virus to approximately 5-7\% of their adult progeny. Vertical transmission did not persist beyond the second generation. Our study of naturally infected $A e$. aegypti found a much higher rate of vertical transmission, with $57.5 \%$ of surviving adult progeny being infected in the first generation. All infected females transmitted the virus to at least some of their $\mathrm{G}_{1}$ offspring. Densovirus infection was maintained for 4 generations in 10 female lines, while transmission to adult progeny was recorded until generation 6 in 4 lines. Filial infection rates in first-generation adult progeny were positively associated with mortality in the same generation. These observations are consistent with those of Barreau and others, ${ }^{9}$ who noted that females that transmitted the virus to their adult offspring also exhibited high mortality rates in their progeny. However, we also found virus transmission to $G_{1}$ progeny in three female lines with mortality rates of less than $10 \%$, suggesting a low virus titer in the mother. Our data indicate the Thai strain densovirus can be persistently maintained over several generations, and may be vertically transmitted by mothers with low virus titers. These findings suggest that vertical transmission may play a more important role in the maintenance of $A T h \mathrm{DNV}$ in nature than it does in other mosquito densoviruses.

We found the prevalence of densovirus infection in field populations of adult Ae. aegypti to vary seasonally, ranging from $6.5 \%$ to $35.1 \%$ over a six-month period. Furthermore, during August and September, when overall prevalence was the lowest, no females $(\mathrm{n}=46)$ tested positive for densovirus. Despite apparently high rates of vertical transmission, horizontal transmission clearly must play a large role in the persistence of $A T h \mathrm{DNV}$ in the field. The relative importance of horizontal and vertical transmission may vary seasonally, in response to variables such as rainfall that may influence the amount of densovirus present in the environment.

Considering that $A T h \mathrm{DNV}$ appears capable of being maintained in field populations of Ae. aegypti and that under laboratory conditions it appears more virulent for Ae. albopictus, it is possible that this virus may be involved in structuring Ae. aegypti and Ae. albopictus populations in nature. Interestingly, Ae. albopictus has been commonly reported to

TABLE 5

Seasonal variation in densovirus infection in natural populations of adult Aedes aegypti from Chachoengsao province, as measured by positive polymerase chain reaction amplification

\begin{tabular}{lccc}
\hline \multicolumn{1}{c}{ Month } & Total infected & Females infected & Males infected \\
\hline April & $22.4 \%(13 / 58)$ & $20.0 \%(3 / 15)$ & $23.3 \%(10 / 43)$ \\
May & $21.6 \%(19 / 88)$ & $11.6 \%(5 / 43)$ & $31.1 \%(14 / 45)$ \\
Jun & $16.3 \%(36 / 221)$ & $20.3 \%(16 / 79)$ & $14.1 \%(20 / 142)$ \\
July & $35.1 \%(108 / 308)$ & $34.4 \%(31 / 90)$ & $35.3 \%(77 / 218)$ \\
August & $6.5 \%(2 / 31)$ & $0 \%(0 / 7)$ & $8.3 \%(2 / 24)$ \\
September & $8.0 \%(11 / 138)$ & $0 \%(0 / 39)$ & $11 / 1 \%(11 / 99)$ \\
Total & $22.4(189 / 844)$ & $20.1 \%(55 / 273)$ & $23.5 \%(134 / 571)$ \\
\hline
\end{tabular}


outcompete Ae. aegypti in the United States, and in certain areas may be replacing it. ${ }^{20-22}$ It has been proposed that a Gregarine parasite may be mediating competition between the two mosquito species in North America. ${ }^{23-25}$ However, similar observations have not been reported in Thailand, where Ae. aegypti numbers have remained high even in areas where the two species share larval habitats. ${ }^{26-28}$ The observed differential mortality between Ae. albopictus and Ae. aegypti when infected with $A T h \mathrm{DNV}$, together with the widespread occurrence of this virus in natural Ae. aegypti populations in Thailand, suggests that the virus may play a role in providing a competitive advantage to Ae. aegypti. Further field studies are needed to determine to what extent $A T h \mathrm{DNV}$ influences competitive outcomes between these two species.

Acknowledgments: We are grateful to Kenneth J. Linthicum for providing mosquito materials. We also thank Samnieng Planuson and Kitti Thienthong for technical assistance.

Financial support: This work was supported by the Thailand Research Fund and the UNDP/World Bank/WHO Special Program for Research and Training in Tropical Diseases.

Authors' addresses: Pattamaporn Kittayapong and Kathy Baisley, Department of Biology, Faculty of Science, Mahidol University, Rama 6 Road, Bangkok 10400, Thailand. Scott L. O'Neill, Section of Vector Biology, Department of Epidemiology and Public Health, Yale University School of Medicine, 60 College Street, New Haven, CT 06520.

Reprint requests: Pattamaporn Kittayapong, Department of Biology, Faculty of Science, Mahidol University, Rama 6 Road, Bangkok 10400, Thailand.

\section{REFERENCES}

1. Tijssen P, Arella M, 1991. Parvoviridae. Structure and reproduction of densonucleosis viruses. Adams JR, Bonami JR, eds. Atlas of Invertebrate Viruses. Boca Raton, FL: CRC Press, 41-53.

2. O'Neill SL, Kittayapong P, Braig HR, Andreadis TG, Gonzalez JP, Tesh RB, 1995. Insect densoviruses may be widespread in mosquito cell lines. J Gen Virol 76: 2067-2074.

3. Afanasiev BN, Galyov EE, Buchatsky LP, Kozlov YV, 1991. Nucleotide sequence and genomic organization of Aedes densonucleosis virus. Virology 185: 323-336.

4. Jousset FX, Barreau C, Boublik Y, Cornet M, 1993. A parvolike virus persistently infecting a C6/36 clone of Aedes albopictus mosquito cell line and pathogenic for Aedes aegypti larvae. Virus Res 29: 99-114.

5. Lebedeva OP, Kuznetsova MA, Zelenko AP, Gudz-Gorban AP, 1973. Investigation of a virus disease of the densonucleosis type in a laboratory cuture of Aedes aegypti. Acta Virol 17: 253-256.

6. Boublik Y, Jousset FX, Bergoin M, 1994. Structure, restriction map and infectivity of the genomic and replicative forms of AaPV DNA. Arch Virol 137: 229-240.

7. Boublik Y, Jousset FX, Bergoin M, 1994. Complete nucleotide sequence and genomic organization of the Aedes albopictus parvovirus $(A a \mathrm{PV})$ pathogenic for Aedes aegypti larvae. Virology 200: 752-763.

8. Gorziglia M, Botero L, Gil F, Esparza J, 1980. Preliminary char- acterization of virus-like particles in a mosquito (Aedes pseudoscutellaris) cell line (Mos. 61). Intervirology 13: 232-240.

9. Barreau C, Jousset FX, Bergoin M, 1997. Venereal and vertical transmission of the Aedes albopictus parvovirus in Aedes aegypti mosquitoes. Am J Trop Med Hyg 57: 126-131.

10. Buchatsky LP, 1989. Densonucleosis of bloodsucking mosquitoes. Dis Aquat Organ 6: 145-150.

11. Barreau C, Jousset FX, Bergoin M, 1996. Pathogenicity of the Aedes albopictus parvovirus (AaPV), a denso-like virus, for Ae. aegypti mosquitoes. J Invertebr Pathol 68: 299-309.

12. O'Neill SL, Giordano R, Colbert AME, Karr TL, Robertson HM, 1992. 16S rRNA phylogenetic analysis of the bacterial endosymbionts associated with cytoplasmic incompatibility in insects. Proc Natl Acad Sci USA 89: 2699-2702.

13. Swofford DL, 1990. PAUP: Phylogenetic Analysis Using Parsimony. Version 3.0. Champaign, IL: Illinois Natural History Survey.

14. Kimura M, 1980. A simple model for estimating evolutionary rates of base substitutions through comparative studies of nucleotide sequences. J Mol Evol 16: 111-120.

15. Felsenstein J, 1993. PHYLIP Phylogeny Inference Package. Version 3.5c. Distributed by the author. Department of Genetics, University of Washington, Seattle.

16. Edman J, Kittayapong K, Linthicum KJ, Scott TW, 1997. Attractant resting boxes for rapid collection and surveillance of Aedes aegypti (L.) inside houses. J Am Mosq Control Assoc 13: 24-27.

17. Kittayapong P, Linthicum KJ, Edman JD, Scott TW, 1997. Further evaluation of the indoor resting boxes for Aedes aegypti surveillance. Dengue Bull 21: 77-83.

18. Buei K, 1983. Pictorial Key to Species, Adult Mosquitoes in Thailand. PPHS-Project-Series No. 3. Bangkok: Ministry of Public Health.

19. Rattanarithikul R, Panthusiri P, 1994. Illustrated Keys to the Medically Important Mosquitoes of Thailand. Bangkok: Wattana Panich Press.

20. O'Meara GF, Evans Jr LF, Gettman AD, Cuda JP, 1995. Spread of Aedes albopictus and decline of Aedes aegypti (Diptera: Culicidae) in Florida. J Med Entomol 32: 554-562.

21. Hobbs JH, Hughes EA, Eichold BH, 1991. Replacement of Aedes aegypti by Aedes albopictus in Mobile, Alabama. J Am Mosq Control Assoc 7: 488-489.

22. Nasci RS, Hare SG, Willis FS, 1989. Interspecific mating between Louisiana strains of Aedes albopictus and Aedes aegypti in the field and laboratory. J Am Mosq Control Assoc 5: 416-421.

23. Blackmore MS, Scoles GA, Craig Jr GB, 1995. Parasitism of Aedes aegypti and Ae. albopictus (Diptera: Culicidae) by Ascogregarina spp. (Apicomplexa: Lecudinidae) in Florida. $J$ Med Entomol 32: 847-852.

24. Craig Jr GB, 1993. The diaspora of the Asian tiger mosquito. McKnight BN, ed. Biological Pollution: The Control and Impact of Invasive Exotic Species. Indianapolis, IN: Indiana Academy of Science, 101-120.

25. Munstermann LE, Wesson DM, 1990. First record of Ascogregarina taiwanensis (Apicomplexa: Lecudinidae) in North American Aedes albopictus. J Am Mosq Control Assoc 6: 235-243.

26. Gratz NG, 1993. Lessons of Aedes aegypti control in Thailand. Med Vet Entomol 7: 1-10.

27. Kittayapong P, Strickman D, 1993. Distribution of containerinhabiting Aedes larva (Dipera: Culicidae) at a dengue focus in Thailand. J Med Entomol 30: 601-606.

28. Eamchan P, Nisalak A, Foy H, Chareonsook OA, 1989. Epidemiology and control of dengue virus infection in Thai villages in 1987. Am J Trop Med Hyg 41: 95-101. 